\title{
Electron Capture Dissociation as Structural Probe for Noncovalent Gas-Phase Protein Assemblies
}

\author{
Rimco B. J. Geels,t,‡ Saskia M. van der Vies,§ Albert J. R. Heck,‡ and Ron M. A. Heeren*,t,‡ \\ FOM Institute for Atomic and Molecular Physics, Kruislaan 407, 1098 SJ Amsterdam, The Netherlands, Department of \\ Biochemistry and Molecular Biology, Vrije Universiteit, De Boelelaan 1083, 1081 HV Amsterdam, The Netherlands, and \\ Department of Biomolecular Mass Spectrometry, Utrecht University, Sorbonnelaan 16, 3584 CA Utrecht, The Netherlands
}

Electron capture dissociation (ECD) of proteins in Fourier transform ion cyclotron resonance mass spectrometry usually leads to charge reduction and backbone-bond cleavage, thereby mostly retaining labile, intramolecular noncovalent interactions. In this report, we evaluate ECD of the 84-kDa noncovalent heptameric gp31 complex and compare this with sustained off-resonance irradiation collisionally activated dissociation (SORI-CAD) of the same protein. Unexpectedly, the $21+$ charge state of the gp31 oligomer exhibits a main ECD pathway resulting in a hexamer and monomer, disrupting labile, intermolecular noncovalent bonds and leaving the backbone intact. Unexpectedly, the charge separation over the two products is highly proportional to molecular weight. This indicates that a major charge redistribution over the subunits of the complex does not take place during ECD, in contrast to the behavior observed when using SORI-CAD. We speculate that the ejected monomer retains more of its original structure in ECD, when compared to SORI-CAD. ECD of lower charge states of gp31 does not lead to dissociation of noncovalent bonds. We hypothesize that the initial gas-phase structure of the $21+$ charge state is significantly different from the lower charge states. These structural differences result in the different reaction pathways when using ECD.

Since the late 1990s, electron capture dissociation ${ }^{1}$ (ECD) has been available as a tool for structural analysis in Fourier transform ion cyclotron resonance mass spectrometry (FT-ICR-MS). To date, ECD is routinely used for top-down sequencing and identification of the posttranslational modifications, such as sulfide bridges, phosphorylation, and methylation. ${ }^{2-7}$ During an ECD experiment,

* To whom correspondence should be addressed. Tel: +31206081234. Fax: +31206684106. E-mail: heeren@amolf.nl.

${ }^{\dagger}$ FOM Institute for Atomic and Molecular Physics.

‡ Utrecht University.

$\S$ Vrije Universiteit.

(1) Zubarev, R. A.; Kelleher, N. L.; McLafferty, F. W. J. Am. Chem. Soc. 1998 , 120, 3265-3266

(2) Kleinnijenhuis, A. J.; Duursma, M. C.; Breukink, E.; Heeren, R. M. A.; Heck, A. J. R. Anal. Chem. 2003, 75, 3219-3225.

(3) McLafferty, F. W. Int. J. Mass Spectrom. 2001, 212, 81-87.

(4) Kjeldsen, F.; Haselmann, K. F.; Budnik, B. A.; Sorensen, E. S.; Zubarev, R. A. Anal. Chem. 2003, 75, 2355-2361.

(5) Ge, Y.; Lawhorn, B. G.; ElNaggar, M.; Strauss, E.; Park, J. H.; Begley, T. P.; McLafferty, F. W. J. Am. Chem. Soc. 2002, 124, 672-678. low-energy electrons are injected into the ICR cell and captured by multiply protonated ions, resulting in the release of approximately $2-6 \mathrm{eV}$ of recombination energy into the ion. ${ }^{1,8}$ Typically, the capture of one or more electrons leads to the rapid dissociation of covalent backbone bonds, resulting in the formation of $\mathrm{c}$ and $z \cdot$ fragment ions. $^{9}$

The ECD process has so far been studied using peptides and proteins with a molecular mass up to $50 \mathrm{kDa}^{5,10}$ but not yet for protein complexes or proteins with a higher molecular mass. There is still an active debate about the exact mechanisms of ECD. ${ }^{8,11-17}$ The early research demonstrated that ECD leads to rapid dissociation of the backbone close to the site of electron capture, not necessarily fragmenting the weakest bonds in the molecule. In line with this observation, ECD has been found to often preserve labile noncovalent bonds, ${ }^{10}$ for instance in cytochrome $c^{18}$ and vancomycin complexed with diacetyl-L-Lys-D-AlaD-Ala. ${ }^{19}$ This makes ECD a potentially useful technique for examining interaction sites in larger, noncovalent protein complexes.

In this report, we use ECD to study the interaction sites in the large gp31 heptameric protein complex from bacteriophage T4. The protein complex has a molecular mass of $84 \mathrm{kDa}$ and consists of seven identical subunits that are noncovalently linked

(6) Iavarone, A. T.; Paech, K.; Williams, E. R. Anal. Chem. 2004, 76, 22312238.

(7) Cournoyer, J. J.; Pittman, J. L.; Ivleva, V. B.; Fallows, E.; Waskell, L.; Costello, C. E.; O'Connor, P. B. Protein Sci. 2005, 14, 452-463.

(8) Breuker, K.; Oh, H. B.; Lin, C.; Carpenter, B. K.; McLafferty, F. W. Proc. Natl. Acad. Sci. U.S.A. 2004, 101, 14011-14016.

(9) Kruger, N. A.; Zubarev, R. A.; Horn, D. M.; McLafferty, F. W. Int. J. Mass Spectrom. 1999, 187, 787-793.

(10) Horn, D. M.; Ge, Y.; McLafferty, F. W. Anal. Chem. 2000, 72, 4778-4784.

(11) Mihalca, R.; Kleinnijenhuis, A. J.; McDonnell, L. A.; Heck, A. J. R.; Heeren, R. M. A. J. Am. Soc. Mass Spectrom. 2004, 15, 1869-1873.

(12) Syrstad, E. A.; Turecek, F. J. Am. Soc. Mass Spectrom. 2005, 16, 208-224.

(13) Zubarev, R. A.; Horn, D. M.; Fridriksson, E. K.; Kelleher, N. L.; Kruger, N A.; Lewis, M. A.; Carpenter, B. K.; McLafferty, F. W. Anal. Chem. 2000 $72,563-573$.

(14) Zubarev, R. A.; Kruger, N. A.; Fridriksson, E. K.; Lewis, M. A.; Horn, D. M.; Carpenter, B. K.; McLafferty, F. W. J. Am. Chem. Soc. 1999, 121, 28572862.

(15) Turecek, F. J. Am. Chem. Soc. 2003, 125, 5954-5963.

(16) Leymarie, N.; Costello, C. E.; O’Connor, P. B. J. Am. Chem. Soc. 2003, 125, 8949-8958.

(17) Sawicka, A.; Skurski, P.; Hudgins, R. R.; Simons, J. J. Phys. Chem. B 2003 , 107, 13505-13511.

(18) Horn, D. M.; Breuker, K.; Frank, A. J.; McLafferty, F. W. J. Am. Chem. Soc. 2001, 123, 9792-9799.

(19) Haselmann, K. F.; Jorgensen, T. J. D.; Budnik, B. A.; Jensen, F.; Zubarev, R. A. Rapid Commun. Mass Spectrom. 2002, 16, 2260-2265. 
together and form a domelike structure with 7-fold symmetry. Gp31 is a co-chaperonin that bacteriophage T4 uses in concert with the GroEL chaperonin of the host bacterium Escherichia coli in order to fold its viral capsid protein. ${ }^{20-25}$ The structure of gp31 is analogous to the $E$. coli co-chaperonin GroES, but is somewhat larger to accommodate the viral capsid protein inside the chaperonin complex. In contrast to the expectations, ECD of the gp31 co-chaperonin leads exclusively to noncovalent bond dissociation for one particular charge state, with a remarkable charge distribution over the two fragments.

\section{EXPERIMENTAL SECTION}

The experiments were performed using a modified Bruker APEX 7.0e FT-ICR-MS equipped with an infinity cell. ${ }^{26}$ Experimental control hard- and software were developed in-house and have been described elsewhere. ${ }^{26}$ Elevated pressure in the source octopole facilitated transfer of the large intact protein complexes into the gas phase and to the ICR cell. Ions were directly gasassisted accumulated inside the ICR cell with typical accumulation times of 10-12 s. Individual charge states of the gp31 heptamer were isolated using SWIFT pulses ${ }^{27}$ and subsequently irradiated with low-energy electrons. ECD settings were optimized for maximum fragmentation. The optimal electron energy was determined to be $\sim 0.3 \mathrm{eV}$. A Heatwave STD134 heated cathode, mounted at the back end of the cell on axis and operated at 1.3 A, 7.5 W, dispensed electrons into the ICR cell. Electrons emitted from the cathode passed through the cell once and were collected on the end plate. Electron irradiation times used in the experiments varied between 2 and 3 s. During ECD and measurement of the ions, trapping plate voltages were lowered to $1.5 \mathrm{~V}$.

In addition to ECD, collision-activated dissociation (CAD) and sustained off-resonance irradiation collision-activated dissociation (SORI-CAD) experiments were also performed. CAD was performed on the ECD products to determine their composition. SORI-CAD was used on the native gp31 heptameric species to compare SORI-CAD pathways with ECD pathways. ${ }^{28}$ SORI-CAD was performed with $\mathrm{a}+1000 \mathrm{~Hz}$ offset and argon collision gas, using procedures described previously. ${ }^{2}$

The gp31 heptamer was purified as described previously. ${ }^{29}$ The protein was buffer exchanged to a $1 \mathrm{mM}$ ammonium acetate buffer, $\mathrm{pH}$ 6.8, by using ultrafiltration filters with a cutoff of 5000

(20) van Duijn, E.; Bakkes, P. J.; Heeren, R. M. A.; van den Heuvell, R. H. H.; van Heerikhuizen, H.; van der Vies, S. M.; Heck, A. J. R. Nat. Methods 2005 , 2, 371-376.

(21) van Duijn, E.; Simmons, D. A.; van den Heuvel, R. H. H.; Bakkes, P. J.; van Heerikhuizen, H.; Heeren, R. M. A.; Robinson, C. V.; van der Vies, S. M.; Heck, A. J. R. J. Am. Chem. Soc. 2006, 128, 4694-4702.

(22) Van der Vies, S. M.; Gatenby, A. A.; Georgopoulos, C. Nature 1994, 368, $654-656$.

(23) Hunt, J. F.; van der Vies, S. M.; Henry, L.; Deisenhofer, J. Cell 1997, 90, $361-371$.

(24) Hunt, J. F.; Weaver, A. J.; Landry, S. J.; Gierasch, L.; Deisenhofer, J. Nature 1996, 379, 37-45.

(25) Sigler, P. B.; Xu, Z. H.; Rye, H. S.; Burston, S. G.; Fenton, W. A.; Horwich, A. L. Annu. Rev. Biochem. 1998, 67, 581-608.

(26) Mize, T. H.; Taban, I.; Duursma, M.; Seynen, M.; Konijnenburg, M.; Vijftigschild, A.; Doornik, C. V.; Rooij, G. V.; Heeren, R. M. A. Int. J. Mass Spectrom. 2004, 235, 243-253.

(27) Guan, S.; Marshall, A. G. Int. J. Mass Spectrom. Ion Processes 1996, $157-$ 158, 5-37.

(28) Fung, Y. M. E.; Duan, L. F.; Chan, T. W. D. Eur. J. Mass Spectrom. 2004, 10, 449-457.

(29) Van der Vies, S. M. Methods Mol. Biol. 2000, 140, 51-61.

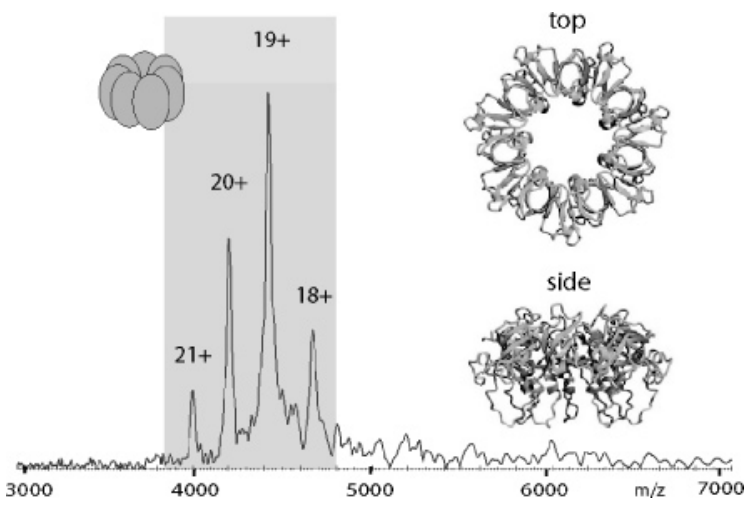

Figure 1. ESI FT-ICR-MS spectrum of the gp31 co-chaperonin complex. Around $4250 \mathrm{~m} / \mathrm{z}$, the charge states 18+ through 21+ of the heptamer are visible. The donut-shaped cartoon represents heptameric gp31. Only the (native) heptameric form of gp31 was present. On the right, the X-ray crystal structure of the heptameric gp31 is shown ${ }^{23}$ (PDB accession code, 1G31).

Da (Millipore, Bedford, MA). Final concentration of gp31 in the spray sample was $13.5 \mu \mathrm{M}$. Protein concentration is given based on the gp31 monomer.

\section{RESULTS AND DISCUSSION}

ECD Leads to Dissociation of Noncovalent Bonds for Specific Charge States of gp31 Heptamers. Figure 1 shows an electrospray ionization (ESI) FT-ICR-MS spectrum of heptameric gp31. Indicated in the figure are the visible charge states. Along with the spectrum, the X-ray crystal structure of gp31 is presented, showing the circular arrangement of the seven noncovalently bound subunits. ${ }^{23}$ Heptamer charge states $18+$ through $21+$ were isolated separately and subjected to ECD under identical experimental conditions. The results are shown in Figure 2.

Remarkably, ECD of gp31 did not result in backbone fragmentation, for all the different charge states that were examined. There are no peaks in the spectra corresponding to backbone fragments. The $\left[\mathrm{M}_{7}+21 \mathrm{H}\right]^{21+}$ species shown in Figure $2 \mathrm{~A}$ exhibited two pathways under ECD. First, multiple electron capture without fragmentation occurs, creating lower charged radical heptamer species. This is reaction I shown in Scheme 1. Second, electron capture is followed by dissociation of noncovalent bonds, creating hexamer and monomer fragments (the observation of pentamer and dimer fragments is discussed below). This is reaction II in Scheme 1. Reaction II may occur either upon first electron capture or after multiple electron captures. The resulting ECD products from reaction II may again be susceptible to subsequent electron capture(s).

The $\left[\mathrm{M}_{7}+20 \mathrm{H}\right]^{20+}$ species, shown in Figure $2 \mathrm{~B}$, also exhibited both pathways, although the pathway following reaction II from Scheme 1 is much less prominent than for the $\left[\mathrm{M}_{7}+21 \mathrm{H}\right]^{21+}$ species, visible only by the $16+$ hexamer peak. ECD of species $\left[\mathrm{M}_{7}+19 \mathrm{H}\right]^{19+}$ and $\left[\mathrm{M}_{7}+18 \mathrm{H}\right]^{18+}$, shown in Figure $2 \mathrm{C}$ and $\mathrm{D}$, only followed reaction I leading to charge reduction. For the latter two precursor ions, reaction I occurred up to seven times before the ion signal disappeared, creating ions of charges down to $12+$ and $11+$, respectively. The release of up to seven times the recombination energy into the molecule thus does not result in any visible dissociation of noncovalent bonds or cleavage of backbone bonds. ECD on large proteins leading to charge 

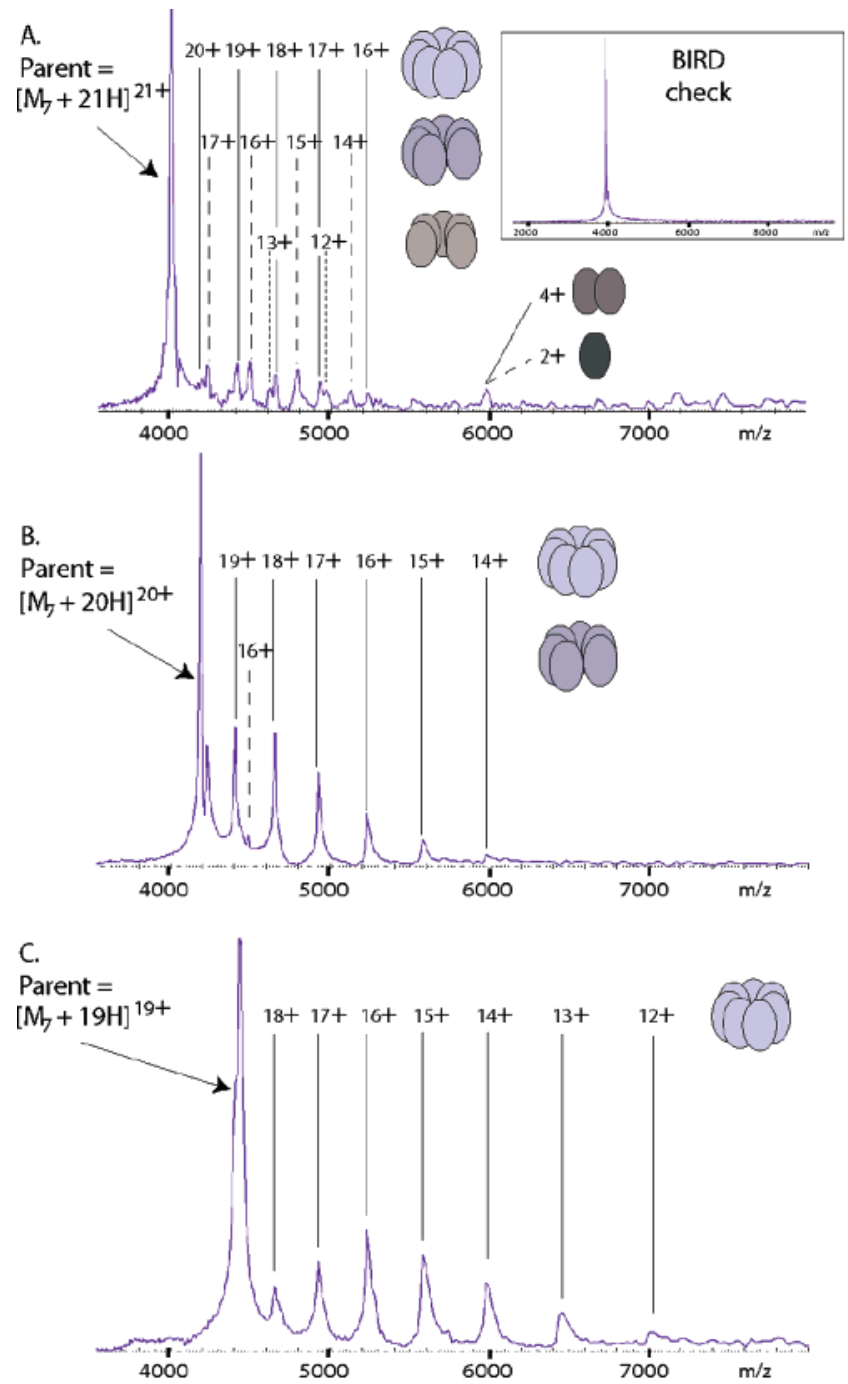

D.

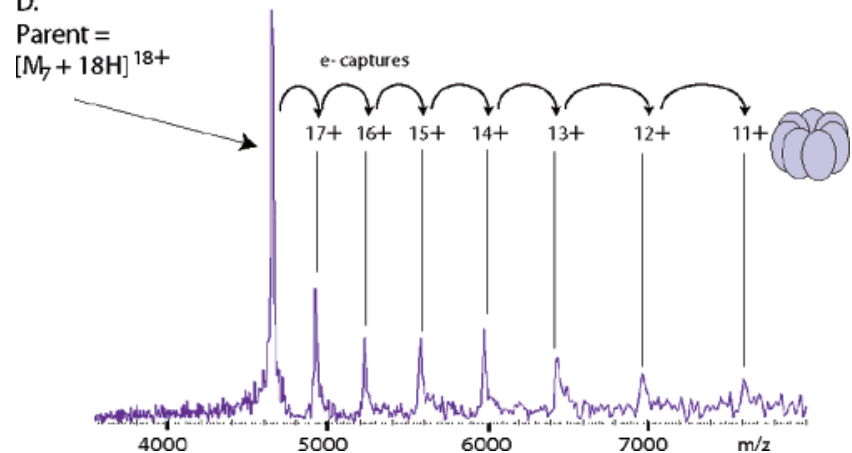

Figure 2. ECD spectra of the different charge states of the intact gp31. The peaks in the spectra are annotated with corresponding charge states and cartoon representations of the oligomeric state. All mass-selected parent ions were subjected to ECD for $3 \mathrm{~s}$. (A) The most abundant fragment ions correspond to lower charged heptamers as well as smaller oligomers, indicating that ECD of $\left[\mathrm{M}_{7}\right.$ $+21 \mathrm{H}]^{21+}$ causes prominent charge reduction and dissociation. (B) $\mathrm{ECD}$ of $\left[\mathrm{M}_{7}+2 \mathrm{OH}\right]^{20+}$ results in prominent charge reduction and minor dissociation. (C, D) ECD of parent species $\left[\mathrm{M}_{7}+19 \mathrm{H}\right]^{19+}$ and $\left[\mathrm{M}_{7}+\right.$ $18 \mathrm{H}]^{18}$ results only in charge-reduced heptamer species. In (D), the subsequent electron captures are also indicated, showing seven subsequent electron captures. The inset in (A) shows a reference spectrum with the electron gun on and electrons repelled from the ICR cell. This spectrum confirms that fragmentation does not occur due to blackbody infrared radiative dissociation from the heated cathode.
Scheme 1. Reaction Pathways of Gp31 21+ upon Activation Using ECD (I and II) or SORI-CAD (III and IV)a

I. $\quad\left[\mathrm{M}_{7}+21 \mathrm{H}\right]^{21+} \stackrel{e^{e} \text { capt. }}{\longrightarrow}\left[\mathrm{M}_{7}+21 \mathrm{H}\right]^{20+}-\mathrm{e}^{\mathrm{e} \text { capt. }}$

II. $\left[\mathrm{M}_{7}+21 \mathrm{H}\right]^{21+} \stackrel{\mathrm{e}^{\mathrm{e}} \text { capt. }}{\longrightarrow}\left[\mathrm{M}_{6}+(20-\mathrm{m}) \mathrm{H}\right]^{(20-\mathrm{m})+}+\left[\mathrm{M}_{1}+\mathrm{mH}\right]^{\mathrm{m+}}+\mathrm{H}$

III. $\left[\mathrm{M}_{7}+21 \mathrm{H}\right]^{21+} \stackrel{\text { SORI-CAD }}{\longrightarrow}\left[\mathrm{M}_{7}+2 \mathrm{OH}\right]^{20+}+\mathrm{H}^{+}$

IV. $\left[\mathrm{M}_{7}+21 \mathrm{H}\right]^{21+} \stackrel{\text { SORI-CAD }}{\longrightarrow}\left[\mathrm{M}_{6}+(21-\mathrm{k}) \mathrm{H}\right]^{(21-\mathrm{k})+}+\left[\mathrm{M}_{1}+\mathrm{kH}\right]^{\mathrm{k}+}$

a (I) Electron capture leads to charge reduction of the complex without fragmentation, creating a radical complex. (II) Electron capture leads to dissociation of the complex creating a hexamer and a monomer. The radical $\mathrm{H}$-atom resides with either the hexamer or the monomer. (III) SORI-CAD leads to charge reduction via ejection of a positive charge. Resolution is not sufficient to ascertain the identity of the ejected fragment. In the reaction this particle is symbolized by $\mathrm{H}^{+}$. (IV) SORI-CAD leads to dissociation of the complex creating a hexamer and a monomer. The charge states of the ejected monomers in reactions II and IV are different. In (II), $m=2-3$ and, in (IV), $k=$ $7-9$.

reduction without fragmentation has been shown before. ${ }^{13,14} \mathrm{ECD}$ leading to dissociation of small, noncovalently bound peptide complexes has also been demonstrated. ${ }^{19}$ However, ECD of large, noncovalently bound protein complexes resulting in dissociation of intermolecular noncovalent bonds has never been shown before to the knowledge of the authors. Moreover, the fact that it only occurs for specific charge states makes this observation even more interesting.

To confirm that dissociation of the noncovalent bonds was not due to thermal activation by blackbody IR photons emitted by the heated cathode, a reference spectrum was obtained (Figure $2 \mathrm{~A}$, inset). This spectrum reveals there was no fragmentation when the heated cathode was on and the electrons were repelled from the ICR cell, revealing that the observed dissociation is truly electron capture induced.

Pentamer Species of gp31 Originate via Dimer Ejection from the Heptamer. Pentamers with charge states $12+$ and $13+$ were seen to be formed in the ECD experiment on the $\left[\mathrm{M}_{7}+\right.$ $21 \mathrm{H}]^{21+}$ ions, as shown in Figure $2 \mathrm{~A}$. They were generated in a one-step reaction analogous to reaction II, but now with the ejection of a dimer instead of a monomer. Subsequent ejection of two monomers can be excluded, because the fragment hexamers from ECD of $\left[\mathrm{M}_{7}+21 \mathrm{H}\right]^{21+}$ were observed not to dissociate further upon electron irradiation, as illustrated in Figure 3A. The $15+$ hexamer fragment from Figure $2 \mathrm{~A}$ was isolated and subjected to a second round of ECD, i.e., $\mathrm{MS}^{3}$. For this species, only reaction I occurred, i.e., charge reduction without dissociation of noncovalent bonds. Isolation and subsequent ECD of the $5975 \mathrm{~m} / z$ fragment peak, as shown in Figure 3B, revealed that this ion signal originated, at least partly, from dimeric ions. This observation confirms that the pentamers from Figure 2A originate through dimer ejection from the heptamer.

ECD Products Have Not Had Backbone Cleavage. As stated previously, backbone fragmentation without dissociation of the fragment from the complex did not occur. Further evidence for this is provided in Figure 4A, which shows the result of resonant $\mathrm{CAD}$ of the $15+$ hexamer fragment that originated from $\mathrm{ECD}$ of $\left[\mathrm{M}_{7}+21 \mathrm{H}\right]^{21+}$. CAD resulted in fragmentation analogous 


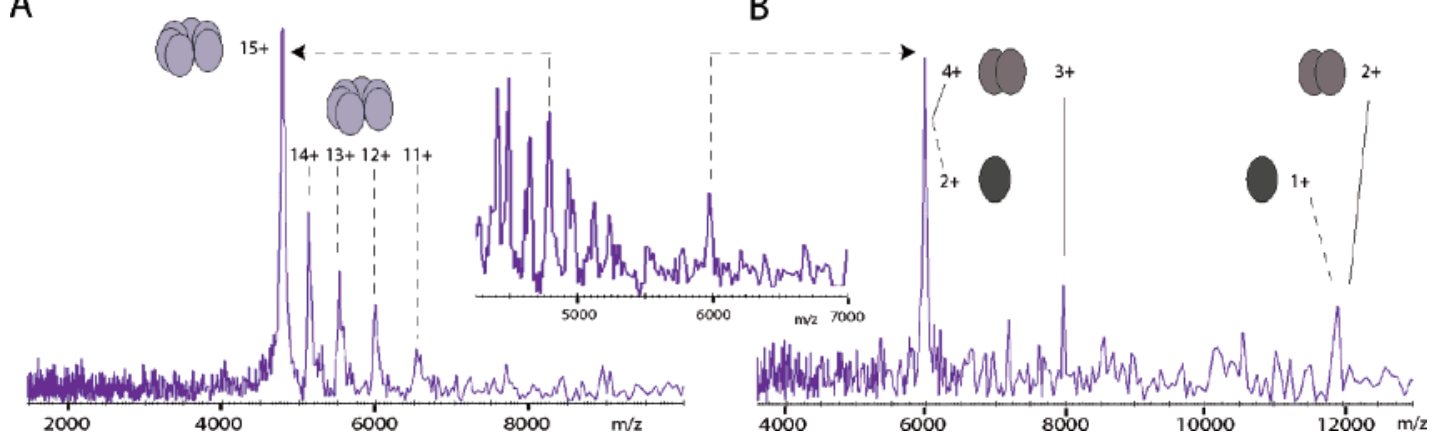

Figure 3. $E C D M S^{3}$ spectra of the $\left[M_{7}+21 \mathrm{H}\right]^{21+}$ gp31 species. In the middle spectrum, the $4250-7000 \mathrm{~m} / \mathrm{z}$ mass region from Figure $2 \mathrm{~A}$ is reproduced, i.e., the first ECD step. From this spectrum, the peaks at 4780 and $5975 \mathrm{~m} / \mathrm{z}$ were mass-selected and examined. (A) ECD of the $15+$ hexamer fragment at $4780 \mathrm{~m} / z$. After ECD the major peak in the spectrum corresponds to the 15+ hexamer "parent". The other peaks present correspond to charge-reduced species of the hexamer. ECD of the hexamer does not result in any dissociation. (B) ECD of the 5975 $\mathrm{m} / \mathrm{z}$ fragment peak. Electron capture resulted in monomers and dimers of low charge states, as indicated. The presence of dimers and monomers after ECD confirms that the "parent" $5975 \mathrm{~m} / \mathrm{z}$ peak consisted of both monomers and dimers.

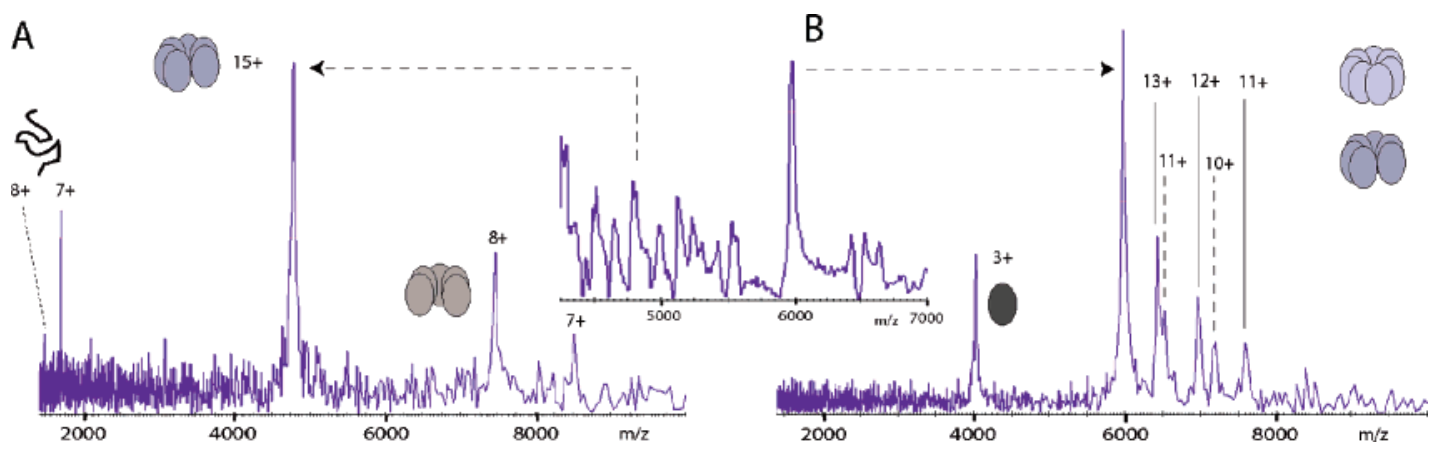

Figure 4. $E C D$ and $C A D M S^{3}$ spectra of the $\left[M_{7}+21 H\right]^{21+}$ gp31 species. The middle spectrum shows a result of $E C D$ of the $[M 7+21 H]^{21+}$ gp31 species, focused on the $4250-7000 \mathrm{~m} / z$ region. Peaks at 4780 and $5975 \mathrm{~m} / z$ were isolated and examined. (A) CAD of the $15+$ hexamer fragment at $4780 \mathrm{~m} / \mathrm{z}$ leading to dissociation of noncovalent bonds to produce a highly charged (i.e., unfolded, as indicated by the cartoon) monomer and corresponding pentamer. There are no peaks corresponding to small backbone fragments. (B) ECD of the $5975 \mathrm{~m} / \mathrm{z}$ product. Heptamers and hexamers are present as ECD products, indicating that the mass-selected "parent" contained the $\left[\mathrm{M}_{7}+21 \mathrm{H}\right]^{14+} \mathrm{species}$. The $3984 \mathrm{~m} / z$ peak is identified as a 3+ (folded) monomer, since a charge-state distribution corresponding to an oligomer is not present. Part of the $\left[\mathrm{M}_{7}+21 \mathrm{H}\right]^{14+}$ thus dissociated into a hexamer and a monomer, confirming the possibility of dissociation after multiple electron capture.

to reaction IV from Scheme 1. All CAD products corresponded to monomers and pentamers without any visible mass losses. From this we infer that the backbone of the $\mathrm{CAD}$ parent, the $15+$ hexamer ECD fragment, was intact. Resonant $\mathrm{CAD}$ on the $2+$ monomer fragment that originated from ECD of $\left[\mathrm{M}_{7}+21 \mathrm{H}\right]^{21+}$ did not result in any $\mathrm{c}$ and $\mathrm{z}$ ions (data not shown), indicating that the backbone of the $2+$ monomer ECD fragment, was intact.

Multiple Electron Capture before Dissociation. We stated that the $\left[\mathrm{M}_{7}+21 \mathrm{H}\right]^{21+}$ complex dissociated either upon the first electron capture or after multiple electron captures. The occurrence of the second option is confirmed by the spectrum in Figure $4 \mathrm{~B}$. In this experiment, the $5975 \mathrm{~m} / z$ fragment peak from the first ECD step was isolated and subjected to a subsequent ECD step, the same as was done to obtain the spectrum in Figure 3B. Contrary to the results in Figure $3 \mathrm{~B}$, in Figure $4 \mathrm{~B}$, mostly heptamers and hexamers are present after ECD. This reveals that the parent peak composition at $5975 \mathrm{~m} / z$ was different for the two experiments. In the second experiment, this peaks consisted mostly of $\left[\mathrm{M}_{7}+21 \mathrm{H}\right]^{14+}$ ions, whereas for the experiment shown in Figure $3 \mathrm{~B}$ it consisted mostly of $2+$ monomers and $4+$ dimers.

The appearance of the $3+$ monomer at $3984 \mathrm{~m} / z$ in Figure $4 \mathrm{~B}$, together with the heptamers and hexamers at higher masses, confirms that the second ECD step dissociated the noncovalent bonds in the $\left[\mathrm{M}_{7}+21 \mathrm{H}\right]^{14+}$ species. Thus, sequential reactions I created the $\left[\mathrm{M}_{7}+21 \mathrm{H}\right]^{14+}$ species, which subsequently dissociated via reaction II.

Comparison of ECD with SORI-CAD Reveals Different Dissociation Mechanics. Dissociation of noncovalent bonds in protein complexes can also be achieved by other activation methods such as (SORI-)CAD or InfraRed multi-photon dissociation..$^{30-33}$ A comparison of the observed fragments created by activation with ECD or with SORI-CAD reveals that charge states of the generated species differ, suggesting that the dissociation mechanism for ECD is different from that in SORI-CAD. In Figure 5 , the $21+$ ECD spectrum from Figure $2 \mathrm{~A}$ is compared with the results obtained from SORI-CAD of that same species. With SORI$\mathrm{CAD}$, two breakdown pathways are identified. In the first pathway, charge reduction of the activated heptamer is observed. After which, the charge-reduced heptamer (now 20+) was no longer activated and did not react further. This behavior corresponds to reaction III in Scheme 1. In the second pathway, the activated

(30) Heck, A. J. R.; van den Heuvel, R. H. H. Mass Spectrom. Rev. 2004, 23, $368-389$.

(31) Sobott, F.; McCammon, M. G.; Robinson, C. V. Int. J. Mass Spectrom. 2003, $230,193-200$

(32) Versluis, C.; van der Staaij, A.; Stokvis, E.; Heck, A. J. R.; de Craene, B. J. Am. Soc. Mass Spectrom. 2001, 12, 329-336.

(33) Laskin, J.; Futrell, J. H. Mass Spectrom. Rev. 2005, 24, 135-167. 


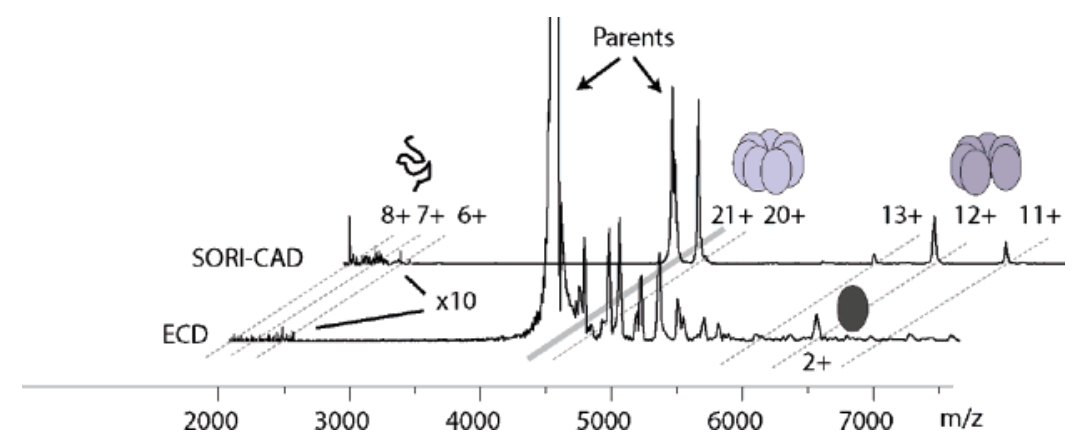

Figure 5. Tandem MS spectra of the gp31 co-chaperonin using two different activation techniques: ECD and SORI-CAD. The ECD spectrum of Figure $2 \mathrm{~A}$ is reproduced here. The lower $\mathrm{m} / \mathrm{z}$ range is multiplied 10 times to show that peaks here are present for SORI-CAD, but are absent with ECD. SORI-CAD resulted in heptamers, hexamers, and monomers of the indicated charge states, showing that both SORI-CAD reactions from Scheme 1 occurred. Even though both ECD and SORI-CAD resulted in dissociation of noncovalent bonds into the same oligomeric species, the charge separation over the fragments was significantly different.

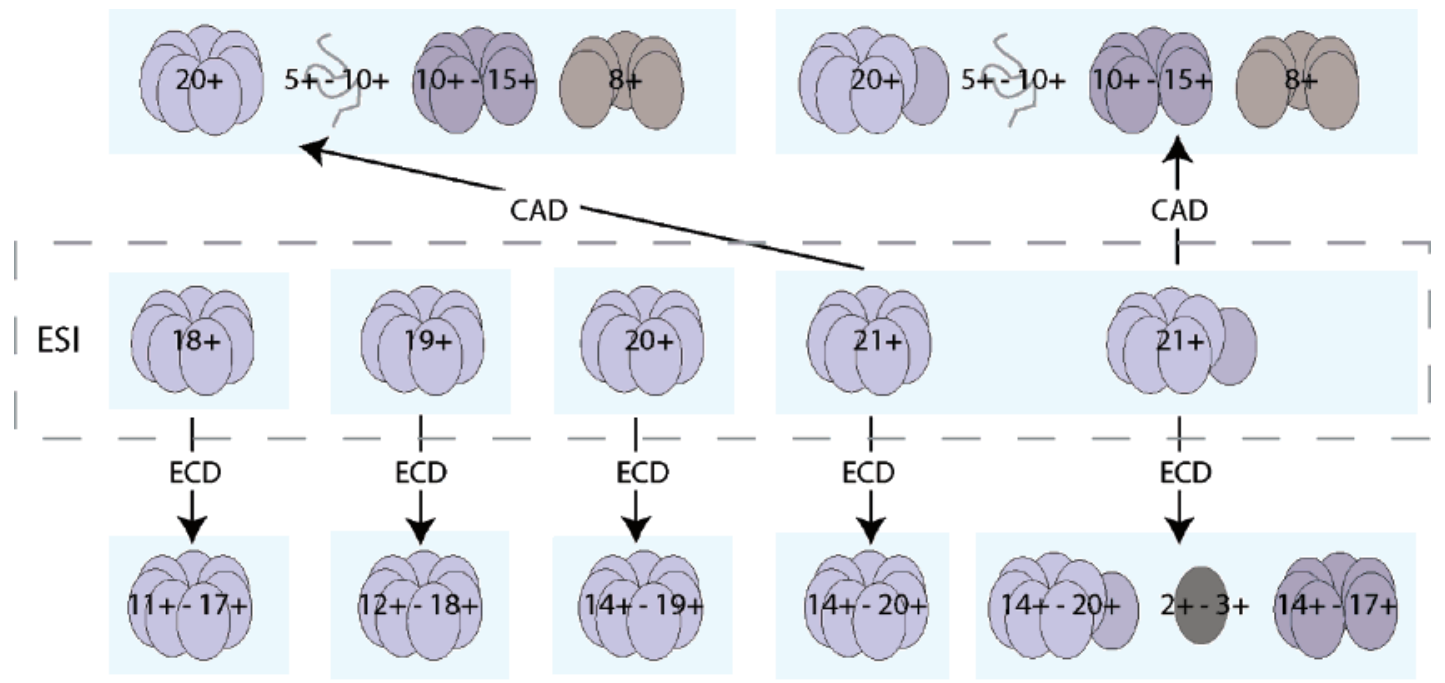

Figure 6. Schematic representation of the hypothesis that different gas-phase structures for the $21+$ charge state of gp31 cause the different pathways observed for ECD. The center of the figure shows the charge states and structures after ESI. These species are subject to ECD (the arrows downward) with the resulting species dependent on the parent structure, or they are subject to SORI-CAD (the arrows upward) with the resulting species not dependent on the parent structure. The scheme also shows that the ejected monomer remains relatively more folded when using ECD than when using SORI-CAD.

complex dissociated into a highly charged monomer and corresponding hexamer. This corresponds to reaction IV from Scheme 1.

In our experiment, ECD leads to symmetric charge separation over the fragments, while SORI-CAD leads to asymmetric charge separation. This disparate charge distribution has been observed numerous times in $\mathrm{CAD}$ experiments of protein complexes. It is believed that Coulombic forces drive the dissociation, which proceeds via the partial unfolding of a single subunit from the complex. While this subunit unfolds, a charge redistribution takes place. When the monomer is sufficiently unfolded and charged, Coulombic repulsion is thought to lead to expulsion of the highly charged monomer. ${ }^{30-32,34-37}$

The charge redistribution to the ejected monomer, as seen with SORI-CAD, is absent in the case of ECD. Therefore, dissociation of noncovalent bonds via ECD is thought not to alter

(34) Jurchen, J. C.; Williams, E. R. J. Am. Chem. Soc. 2003, 125, 2817-2826. (35) Jurchen, J. C.; Garcia, D. E.; Williams, E. R. J. Am. Soc. Mass Spectrom. 2004, 15, 1408-1415.

(36) Sobott, F.; Robinson, C. V. Curr. Opin. Struct. Biol. 2002, 12, 729-734.

(37) Pinkse, M. W. H.; Maier, C. S.; Kim, J. I.; Oh, B. H.; Heck, A. J. R. J. Mass Spectrom. 2003, 38, 315-320. the underlying structure of the monomer. The monomer dissociation from the complex using ECD is rapid relative to dissociation with SORI-CAD, otherwise the Coulomb forces would redistribute charges to the monomer during ejection. The observed rapid dissociation with ECD can be interpreted as supporting the nonergodic dissociation mechanism for ECD, but the data are not conclusive on this matter.

Gas-Phase Structure Determines ECD Pathway. In summary, we have shown the dissociation of species $\left[\mathrm{M}_{7}+21 \mathrm{H}\right]^{21+}$, $\left[\mathrm{M}_{7}+20 \mathrm{H}\right]^{20+}$, and $\left[\mathrm{M}_{7}+21 \mathrm{H}\right]^{14+}$ upon electron capture, while no dissociation was observed for the $\left[\mathrm{M}_{7}+19 \mathrm{H}\right]^{19+},\left[\mathrm{M}_{7}+\right.$ $18 \mathrm{H}]^{18+},\left[\mathrm{M}_{7}+19 \mathrm{H}\right]^{14+}$, and $\left[\mathrm{M}_{7}+18 \mathrm{H}\right]^{14+}$ species, as visible in Figure 2C and D. We hypothesize that different gas-phase structures of the various gp31 heptamer species determine the behavior with ECD. Variations in ECD behavior between proteins have been attributed to conformational differences. ${ }^{11,38}$ It has been shown that gas-phase structures of a single protein differ between specific charge states and even differ for the same charge state. ${ }^{39}$ Increasing Coulomb repulsion through an increase of the number

(38) Breuker, K.; Oh, H. B.; Horn, D. M.; Cerda, B. A.; McLafferty, F. W. J. Am. Chem. Soc. 2002, 124, 6407-6420.

Analytical Chemistry, Vol. 78, No. 20, October 15, 2006 
of charges on the ion during the evaporation process induces conformational changes. It is thought that for heptamer charge states up to $20+$ the Coulomb repulsion is not large enough to inflict serious structural deformations from the 7-fold symmetric ring arrangement. However, the $21+$ heptamer experiences significant Coulomb repulsion, and at least part of the gas-phase population has undergone a conformational change away from the 7-fold symmetry. As a result, we conclude that the overall structure exhibits a deformation that makes it susceptible to dissociation by ECD.

One can imagine that, during ECD, electron capture at or close to the structurally deformed site will lead to rapid dissociation of the complex, whereas electron capture at sites away from the deformation will only lead to charge reduction. The actual dissociation event is still caused by a single electron capture. To account for the dissociation of the $\left[\mathrm{M}_{7}+21 \mathrm{H}\right]^{14+}$ species with ECD in Figure 4B, we postulate that charge reduction by electron capture in the gas phase will not lead to a structural rearrangement back to the 7-fold symmetric ring but will leave the ion susceptible to dissociation upon a next electron capture.

The analytical results and the hypothesis on the influence of conformation are schematically summarized in Figure 6. The structural deformation for the $21+$ charge state is visualized as a dislocation of one subunit. Contrary to ECD, for SORI-CAD, the complex is slowly heated and the original gas-phase structure is not relevant for the eventual breakdown pathway.

(39) McLafferty, F. W.; Guan, Z. Q.; Haupts, U.; Wood, T. D.; Kelleher, N. L. J. Am. Chem. Soc. 1998, 120, 4732-4740.

\section{CONCLUSION}

ECD of the noncovalent gp31 co-chaperonin complex does not lead to cleavage of covalent backbone bonds as has been observed for monomeric proteins, but instead to rapid disassembly of the complex for which multiple noncovalent intermolecular interactions have to be broken. The backbone of the subunits remains intact and the ejected subunit does not unfold during dissociation from the complex. These observations are very different from what has been reported for ECD on smaller, monomeric proteins. We conclude that the $21+$ charge state of the complex has a gasphase structure that is different from that of the lower charge states, allowing ECD to dissociate noncovalent bonds. This conclusion implies that the underlying differences in gas-phase structure lead to the observed different dissociation pathways for $\mathrm{ECD}$, but not for (SORI-)CAD. This thus indicates that the conformation of a protein plays a crucial role in ECD.

\section{ACKNOWLEDGMENT}

We thank Esther van Duijn for kindly providing the protein samples. This research is part of the research program of the "Stichting voor Fundamenteel Onderzoek der Materie (FOM)", which is financially supported by the "Nederlandse organisatie voor Wetenschappelijk Onderzoek (NWO)”. This work is part of the FOM/ALW program "Physical Biology II" under project 01FB12-1.

Received for review May 24, 2006. Accepted August 11, 2006.

AC060960P 